\title{
Progress toward a capacitively mediated CNOT between two charge qubits in $\mathrm{Si} / \mathrm{SiGe}$
}

\author{
E. R. MacQuarrie ${ }^{1}{ }^{1}$, Samuel F. Neyens ${ }^{1}$, J. P. Dodson ${ }^{1}$, J. Corrigan ${ }^{1}$, Brandur Thorgrimsson ${ }^{1}$, Nathan Holman ${ }^{1}$, M. Palma ${ }^{1}$, L. F. Edge ${ }^{2}$, \\ Mark Friesen (iD) ${ }^{1}$, S. N. Coppersmith (iD ${ }^{1,3}$ and M. A. Eriksson ${ }^{1 凶}$
}

Fast operations, an easily tunable Hamiltonian, and a straightforward two-qubit interaction make charge qubits a useful tool for benchmarking device performance and exploring two-qubit dynamics. Here, we tune a linear chain of four Si/SiGe quantum dots to host two double dot charge qubits. Using the capacitance between the double dots to mediate a strong two-qubit interaction, we simultaneously drive coherent transitions to generate correlations between the qubits. We then sequentially pulse the qubits to drive one qubit conditionally on the state of the other. We find that a conditional $\pi$-rotation can be driven in just 74 ps with a modest fidelity demonstrating the possibility of two-qubit operations with a $13.5 \mathrm{GHz}$ clockspeed.

npj Quantum Information (2020)6:81 ; https://doi.org/10.1038/s41534-020-00314-w

\section{INTRODUCTION}

With charge, valley, and spin degrees of freedom, quantum dots in silicon are promising hosts of many different types of qubits. Using the electronic spin state as the logical basis has enabled high fidelity single-qubit operations ${ }^{1}$ and demonstrations of twoqubit gates $^{2-9}$. To date, two-qubit gates in Si quantum dots have been mediated by a spin-spin exchange interaction or by coupling via a superconducting resonator ${ }^{10}$.

Alternatively, a capacitive interaction can be used to coherently couple neighboring double dot qubits using the electronic charge degree of freedom. Capacitive coupling has been used to perform fast two-qubit operations in charge qubits ${ }^{11}$ and singlet-triplet qubits $^{12,13}$ in GaAs quantum dot devices.

In Si-based quantum dot devices, a strong ${ }^{14}$ and tunable ${ }^{15}$ capacitive interaction between double dots has been demonstrated and used to perform qubit control conditionally on the state of a classical two level system ${ }^{16}$. The strength of this interaction makes it a promising candidate for coupling qubits that have a tunable charge dipole moment such as the quantum dot hybrid qubit $(\mathrm{QDHQ})^{17-19}$. Fast, multi-qubit control presents a number of challenges, however, such as pulse synchronization across a device. Here, we use capacitively-coupled charge qubits to explore these challenges by measuring correlated oscillations between two simultaneously-driven qubits, by using those dynamics to synchronize our multi-qubit control channels, and by using this interaction to drive a fast (74 ps) conditional $\pi$-rotation.

\section{RESULTS}

Device details

To perform capacitively-coupled two-qubit measurements, we fabricate a linear chain of four quantum dots using an overlapping-aluminum gate architecture (Fig. 1a) ${ }^{14,15,20,21}$. The fabrication details for this device have been reported elsewhere ${ }^{15}$. Measurements are performed in an Oxford Triton 400 dilution refrigerator with a $\sim 15 \mathrm{mK}$ mixing chamber temperature. We tune the device to host two tunnel-coupled double dots, each nominally residing in the $(1,0)-(0,1)$ charge configuration (Fig. 1b). In all measurements reported here, the double dot electron temperature was $T_{0}^{\text {elec }}=228 \mathrm{mK}$ and the charge reservoir temperature was $T_{0}^{\text {res }}=321 \mathrm{mK}$ (see Supplementary Note 1).

Two additional quantum dots are formed on the bottom half of the device to enable charge sensor readout of the qubit states. The current through the left charge sensor feeds into a room temperature current preamplifier and the resulting voltage is measured with a lock-in amplifier. The right charge sensor current is amplified at the mixing chamber of our dilution unit using a home-built two-stage cryogenic amplifier ${ }^{22}$. It is then amplified again at room temperature, and the resulting voltage is measured with a lock-in amplifier. While the right charge sensor only responds to the right double dot (RDD), during qubit operations the left charge sensor measures both the RDD and the left double dot (LDD). Both charge sensors are used in the measurements that follow. As detailed in Methods section, appropriate normalization measurements allow us to subtract the calibrated RDD signal from the left charge sensor data, enabling independent measurement of the two qubits.

Qubit initialization, control, and latched readout

In our device, each double dot has an outer dot that neighbors a charge reservoir and an inner dot that is isolated from any reservoir. During qubit operations, we initialize each charge qubit at a large detuning $\varepsilon_{1}$ where one electron localizes into the qubit's outer dot $\left(\left|\psi_{0}\right\rangle=|L\rangle\right.$ for the LDD; $\left|\psi_{0}\right\rangle=|R\rangle$ for the RDD). Singlequbit operations are described well by a charge qubit Hamiltonian

$H_{1 \mathrm{Q}}=\frac{\varepsilon}{2} \sigma_{z}+t_{\mathrm{c}} \sigma_{x}$

where $\varepsilon$ is the double dot detuning, $t_{\mathrm{c}}$ is the tunnel coupling, and $\sigma_{x}, \sigma_{z}$ are the standard Pauli operators in the position basis $\{L, R\}$. As discussed in Supplementary Note 3 , we have neglected the valley excited states that can be seen in Si/SiGe quantum dot qubits.

To perform quantum control, we apply a fast dc pulse to move the system to $\varepsilon=0$. This rapid pulse non-adiabatically changes the 

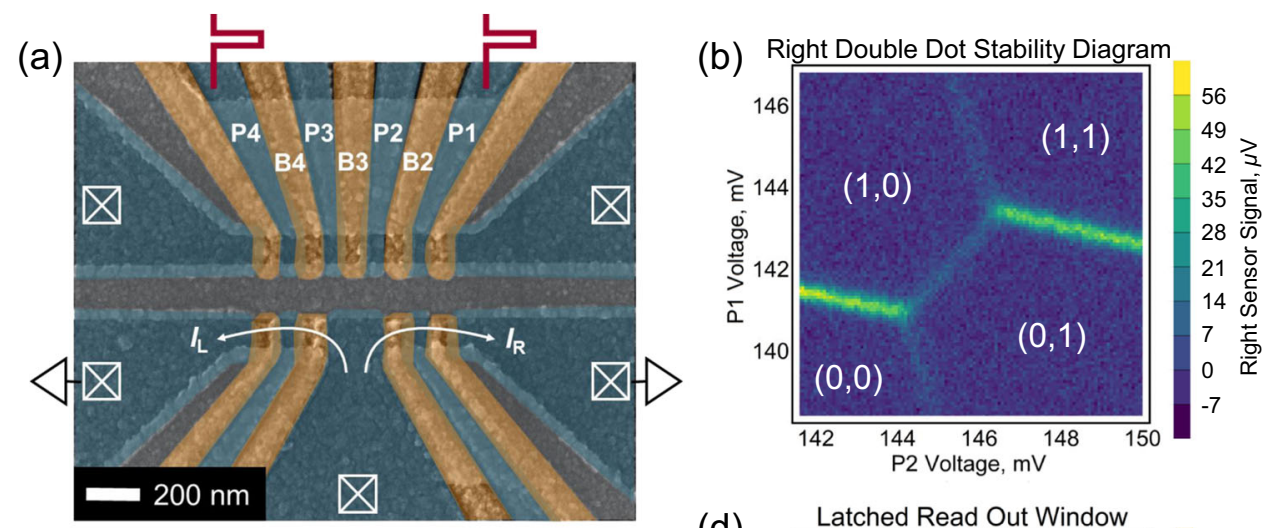

(c)
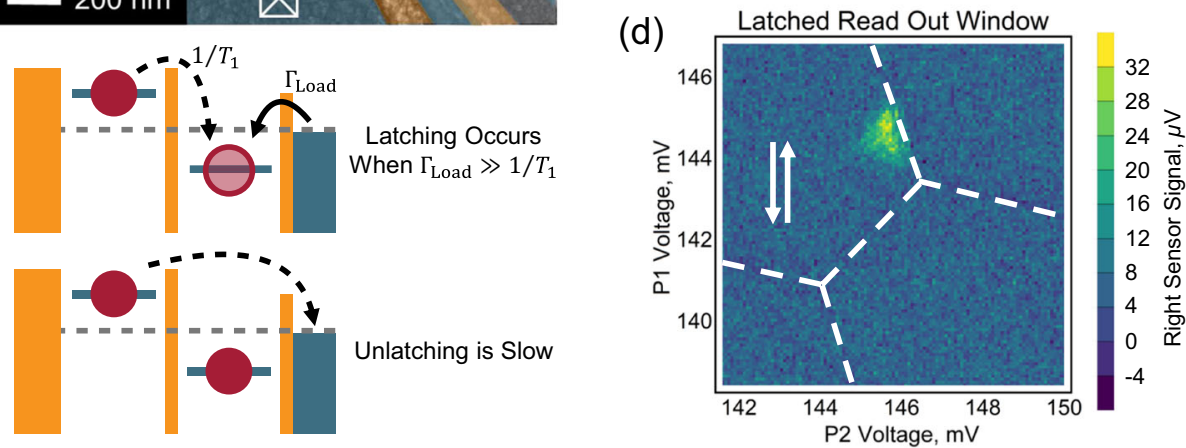

Fig. 1 Device and measurement details. a False-color scanning electron micrograph of a device nominally identical to the one measured here. Gates P1 and P4 are used for fast dc control as described in Supplementary Note 2. The scale bar indicates $200 \mathrm{~nm}$. b Stability diagram of the right double dot tuned to the nominal $(1,0)-(0,1)$ charge configuration. c Schematic depiction of latched state readout for the right double dot with a charge reservoir to the right and a hard-wall potential on the left. Latched readout projects the excited charge qubit state into a $(1,1)$ charge configuration when the tunnel rate from the charge reservoir $\Gamma_{\text {Load }}$ exceeds the charge qubit relaxation rate $1 / T_{1}$. d Stability diagram in $\mathbf{b}$ with fast dc control pulses applied to P1. The bright triangular region indicates the latched readout window, and the white arrows illustrate the applied dc pulse.

qubit Hamiltonian to generate $\sigma_{x}$ rotations at a rate of $2 t_{c}$. These rotations persist until the detuning is moved back to $\varepsilon_{1}$. If some fraction of the electron remains in the inner dot at the end of the coherent evolution, then there is nonzero probability that a second electron will tunnel from the reservoir into the outer dot before the qubit relaxes into $\left|\psi_{0}\right\rangle$. When this occurs, the qubit is projected into the $(1,1)$ charge configuration and remains there until a co-tunneling process reinitializes the qubit into $\left|\psi_{0}\right\rangle$, providing a latched-state readout process ${ }^{23,24}$.

Using the metastable $(1,1)$ charge configuration for latchedstate readout provides two advantages. First, when the qubit enters the latched state, a second electron is added to the double dot system. This produces a larger shift in the charge sensor current than the mere relocation of a single electron. Secondly, this change in charge configuration persists for a much longer time because the co-tunneling process needed for reinitialization is generally much slower ( $T_{\text {Latch }}>100 \mathrm{~ns}$ ) than charge qubit relaxation $\left(T_{1}<10 \mathrm{~ns}\right.$ in this device-see Supplementary Note 4 for measurement details). Both of these mechanisms increase the signal generated by our qubit measurements.

To maximize the probability that a driven state becomes latched, we tune the tunnel rate between the reservoir and the outer dot to be much larger than the charge relaxation rate between the two dots $\left(\Gamma_{\text {Load }} \gg 1 / T_{1}\right)$. Figure $1 \mathrm{c}$ provides a schematic representation of this latched measurement strategy for the RDD, and Fig. 1d shows the latched state readout window that appears when dc pulses are applied to the stability diagram in Fig. 1b. This measurement was performed by shuttering our control pulses at a fixed repetition rate, locking in to the presence and absence of control pulses, and measuring the time-averaged charge sensor response. All qubit data reported here were measured with this latched-state, time-averaged technique.
Single-qubit measurements

With each qubit tuned to the nominal $(1,0)-(0,1)$ charge configuration, we use dc control pulses to perform single-qubit Ramsey measurements of the qubit inhomogeneous dephasing times $T_{2}^{*}$. The pulse sequence (Fig. 2a) begins with initialization at large detuning $\varepsilon_{1}$. A sudden dc-shift to $\varepsilon=0$ turns on $\sigma_{x}$ rotations in the $\{L, R\}$ basis. After a $(n+1) \pi / 2$ rotation, we apply a second dc-shift, moving to nonzero detuning and adding a $\sigma_{z}$ component to the Hamiltonian to start phase accumulation. Returning to $\varepsilon=0$ allows us to perform a second $(n+1) \pi / 2$ rotation, projecting the accumulated phase onto the $z$-axis of the $\{L, R\}$ Bloch sphere. Finally, moving back to $\varepsilon_{1}$ for latched state readout maps the charge qubit coherence onto the measured charge sensor current $^{25}$.

The Ramsey data for the LDD and RDD are shown in Fig. 2b, $c$, respectively. Both qubits display coherent behavior. By extracting the frequency of the Ramsey fringes as a function of detuning, we map the dispersion of our qubits and confirm that Eq. (1) appropriately describes each system. At large detuning $(\varepsilon / h>30$ $\mathrm{GHz}$ ), however, the RDD dispersion begins to deviate from the expected charge qubit behavior. This could be due to timing artifacts in our control hardware as the rotation speed surpasses the 40 ps rise time of our waveform generator. Alternatively, this could be evidence of a low-lying valley state generating additional curvature in the dispersion near $\varepsilon=0^{26}$.

The LDD Ramsey fringes lose all visibility for free evolution at $\varepsilon>$ 0 . This could be due to imperfect pulse edges creating unintentional adiabaticity. Such an effect would have been more apparent in the LDD than in the RDD due to the larger tunnel coupling $\left(t_{c}^{\mathrm{L}} / h=9.9 \mathrm{GHz}\right.$ versus $\left.t_{c}^{\mathrm{R}} / h=5.0 \mathrm{GHz}\right)$ requiring faster rise times for true nonadiabatic control. 
(a)

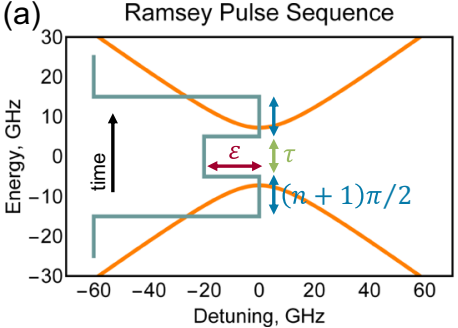

(b) Left Double Dot Ramsey Measurement

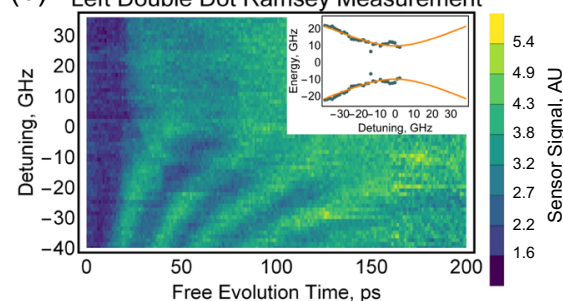

(c) Right Double Dot Ramsey Measurement

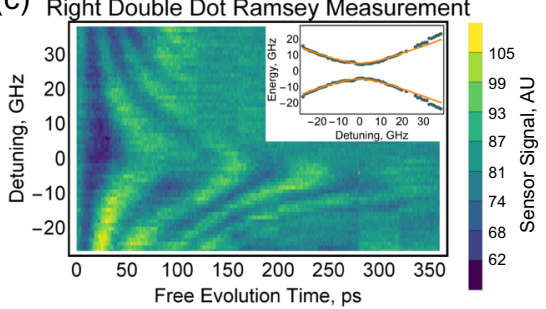

Fig. 2 Single qubit dispersions. a Dispersion (orange) and pulse sequence (cyan) for measuring Ramsey oscillations at a detuning $\varepsilon$ for a free evolution time $\tau$. b, $\mathbf{c}$ Ramsey oscillations measured in $\mathbf{b}$ left and $\mathbf{c}$ right double dots. The extracted charge qubit dispersions with $t_{c}^{\mathrm{L}} / h=9.9$ $\mathrm{GHz}$ and $t_{\mathrm{c}}^{\mathrm{R}} / h=5.0 \mathrm{GHz}$ are shown in the insets.

For large detunings, the qubit dispersion is approximately linear in $\varepsilon$. Assuming non-Markovian detuning noise dominates the dephasing $^{27}$, we can fit the decaying coherence to a Gaussian envelope $e^{-t^{2} / T_{2}^{* 2}}$ and find that for large detunings $T_{2}^{*}=80 \pm 20 \mathrm{ps}$ and $109 \pm 6 \mathrm{ps}$ for the LDD and RDD, respectively. These dephasing times can be explained by quasistatic detuning noise with standard deviations given by $\sigma_{\varepsilon}=h / \sqrt{2} \pi T_{2}^{*}$ where $h$ is Planck's constant. For the LDD and RDD, we find comparable values of $12 \pm 4 \mu \mathrm{eV}$ and $8.5 \pm 0.5 \mu \mathrm{eV}$, respectively (additional details in the Supplementary Note 5).

\section{Correlated Oscillations}

The two qubits in our device are capacitively coupled with a gatevoltage tunable coupling coefficient $g^{15}$. In the two-qubit position basis $\{L L, L R, R L, R R\}$, the Hamiltonian describing this coupled system can be written as ${ }^{11}$

$$
\begin{aligned}
H_{2 \mathrm{Q}}= & \frac{\varepsilon_{\mathrm{L}}}{2} \sigma_{z} \otimes I+t_{\mathrm{c}}^{\mathrm{L}} \sigma_{x} \otimes I+\frac{\varepsilon_{\mathrm{R}}}{2} I \otimes \sigma_{z} \\
& +t_{\mathrm{c}}^{\mathrm{R}} I \otimes \sigma_{x}+\frac{g}{4}\left(I-\sigma_{z}\right) \otimes\left(I-\sigma_{z}\right)
\end{aligned}
$$

where $\varepsilon_{\mathrm{L}}\left(\varepsilon_{\mathrm{R}}\right)$ and $t_{\mathrm{c}}^{\mathrm{L}}\left(t_{\mathrm{c}}^{\mathrm{R}}\right)$ are the detuning and tunnel coupling in the LDD (RDD) and $l$ is the identity matrix. The $\sigma_{z} \otimes \sigma_{z}$ nature of the capacitive interaction generates a detuning offset in one qubit conditionally on the state of the other (Fig. 3a, b). This capacitive interaction can be used to build state correlations between the two qubits, which we will use to synchronize one qubit's control pulses with those of the other. We note that the latched state readout described above also creates a capacitive shift upon projection into the latched state due to the introduction of an additional charge into the system. This shift is of comparable magnitude to the coherent two-qubit interaction but only appears after a qubit has been pulsed into its readout window (details in Supplementary Note 6).

In order to observe such correlations, we first tune a Hamiltonian with $t_{c}^{\mathrm{L}} / h=4.2, t_{c}^{\mathrm{R}} / h=3.3$, and $g / h=15.3 \mathrm{GHz}$. We then pulse the RDD to its anti-crossing at $\varepsilon_{R}=0$. A time $\Delta$ thereafter, we pulse the LDD to $\varepsilon_{\mathrm{L}}=g$, the location of its unshifted anti-crossing. The two qubits then simultaneously evolve according to the two-qubit Hamiltonian $H_{2 Q}$. After an evolution time $\tau_{\mathrm{L}}$ $\left(\tau_{\mathrm{R}}\right)$, we pulse the LDD (RDD) into its readout window for projection into the latched state. Because the latched readout state produces a capacitive shift of similar magnitude to the twoqubit interaction, the RDD (LDD) then continues evolving conditionally on the projected state of the LDD (RDD). By independently varying $\tau_{L}$ and $\tau_{R}$ as shown in Fig. $3 c$, d, we can then observe coherent two qubit dynamics along the diagonal of Fig. 3c, d where both qubits evolve at their respective anticrossings. Away from that diagonal, we observe correlated two qubit evolution since one qubit has been conditionally projected into its latched state for some portion of the measurement. In Fig. $3 \mathrm{~d}$, for instance, when $\tau_{L}<\tau_{R}-\Delta$ the RDD continues evolving after the LDD has been pulsed to its readout window. If the LDD was projected into its latched state, the resulting capacitive shift prevents further oscillations in the RDD. Otherwise, the RDD continues to oscillate with $\tau_{\mathrm{R}}$. Importantly, by using the abrupt change in charge dynamics along the diagonal of Fig. 3 as feedback, we are able to sync our fast dc pulses at the mixing chamber to within $\sim 80$ ps.

As shown in Fig. 3e, $\mathrm{f}$, we recreate the measured two-qubit evolution by numerically solving the von Neumann equation using the Hamiltonian presented in Eq. (2). Dephasing from charge noise is included by convolving this simulation with perturbations to both $\varepsilon_{\mathrm{L}}$ and $\varepsilon_{\mathrm{R}}$ (i.e. $\varepsilon_{i} \rightarrow \varepsilon_{i}+\delta \varepsilon_{i}$ ). We assume these perturbations follow Gaussian distributions with standard deviations given by $\sigma_{\varepsilon}=12$ and $8.5 \mu \mathrm{eV}$, respectively. Notably, the only free parameter in this simulation is the fixed offset between the rising edges of the pulses, which we fix to $\Delta=150 \mathrm{ps}$. Additional simulation details are provided in Supplementary Note 8.

\section{Towards a two-qubit gate}

The capacitive interaction can also be used to drive one qubit conditionally on the state of the other as has been demonstrated experimentally in GaAs charge qubits ${ }^{11}$ and proposed theoretically in Si/SiGe QDHQs ${ }^{19}$. To demonstrate conditional rotations, we designate the LDD the control qubit and the RDD the target. We shift the control qubit to $\varepsilon_{\mathrm{L}}=g$ (the location of the LDD anticrossing for the initialized state $|L R\rangle)$, allow it to evolve for some time $\tau_{L}$, and then shift it to a large detuning $\varepsilon_{\text {idle }}$ that lies outside the readout window. The addition of this idle step delays the conditional projection of the control qubit into its latched state, ensuring that the control qubit remains within its computational basis during target qubit operations. While the control qubit is idling, the target qubit is pulsed to $\varepsilon_{R}=0$ and is conditionally driven dependent on the state populations of the control qubit. This pulse on the target qubit constitutes our conditional driving. Both qubits are then moved into their readout windows for latched-state measurement. We note that the control qubit dephases during its idle period $\left(T_{2}^{*}=80 \pm 20 \mathrm{ps}\right.$ at $\left.\varepsilon_{\text {idle }}\right)$, but because dephasing does not alter qubit state populations, this does not affect the target qubit evolution. The results of this measurement generate the patchwork pattern shown in Fig. 4a, which is a hallmark of conditional evolution.

Next, we characterize the fidelity of a conditional $\pi$-rotation at a tuning where $t_{\mathrm{c}}^{\mathrm{L}} / h=7.2, t_{\mathrm{c}}^{\mathrm{R}} / h=5.4$, and $g / h=28 \mathrm{GHz}$ (Supplementary Note 6). Our latched-state measurement technique provides the time-averaged values of $\left\langle\sigma_{z} \otimes I\right\rangle$ and $\left\langle I \otimes \sigma_{z}\right\rangle$. Without joint single shot readout or a verified, high fidelity twoqubit gate, this is not enough information to perform two-qubit tomography ${ }^{28}$, so we restrict our analysis to input states for which both qubits are expected to evolve into single-qubit eigenstates. For these inputs, we can assume the resulting two-qubit state is separable and our readout provides the appropriate populations for construction of the truth table $M_{\exp }$ describing our conditional operation. 

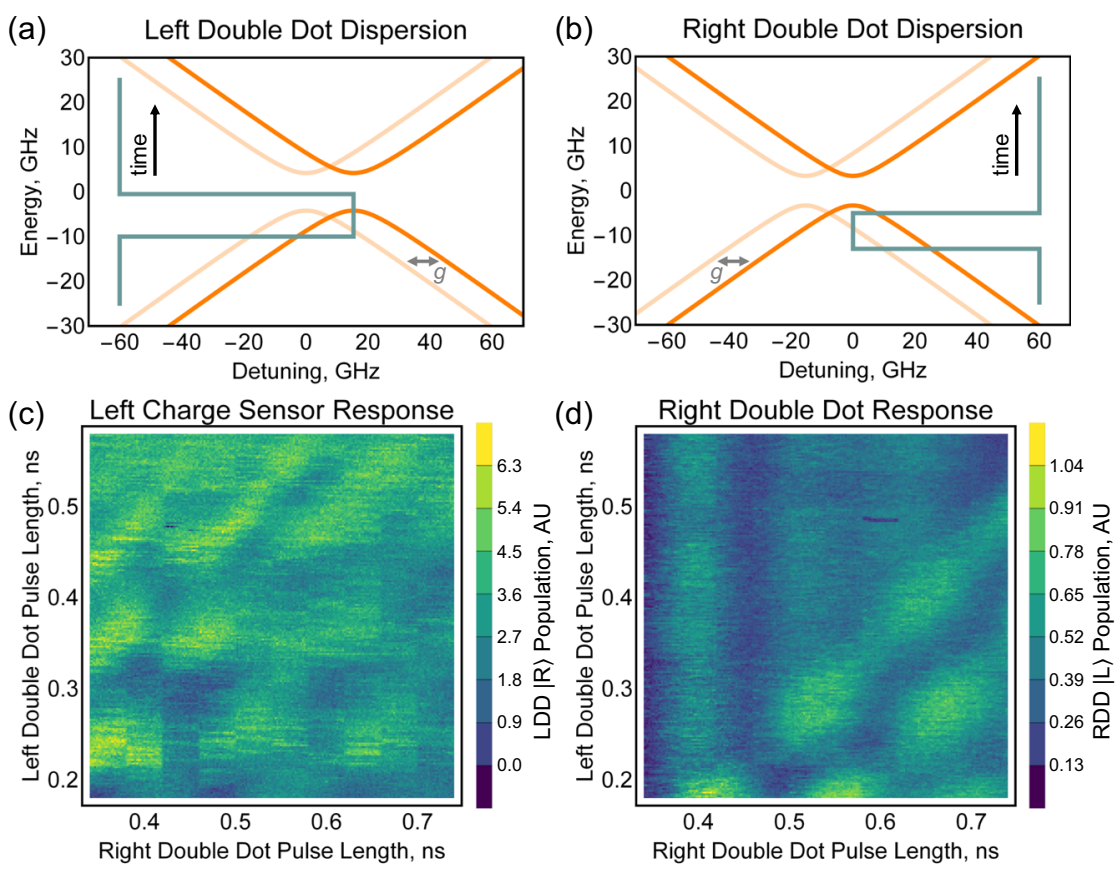

(e) Simulated Left Double Dot Response

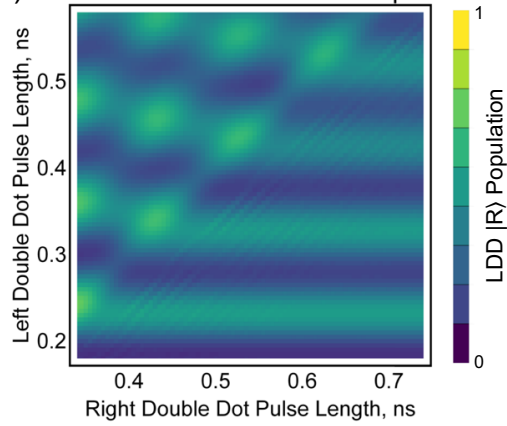

(f) Simulated Right Double Dot Response

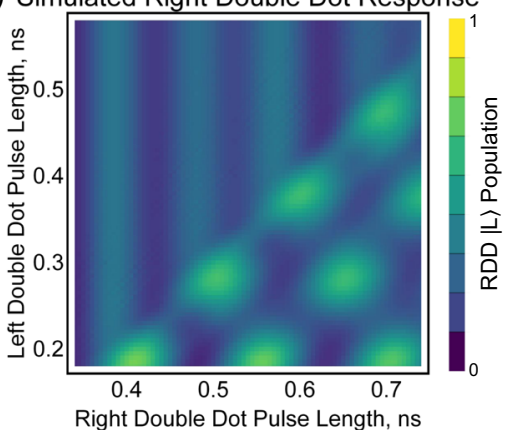

Fig. 3 Correlated qubit evolution. a, b Dispersions (orange) and pulse sequences (cyan) for simultaneously driving two charge qubits. Both the capacitively-shifted (light orange) and unshifted (dark orange) dispersions are shown. $\mathbf{c}$, $\mathbf{d}$ Measured two-qubit response to simultaneous driving. In c, charge sensor crosstalk has been subtracted as described in the Methods section, and the black pixels lie outside the range of the plotted color scale. In d, a jump in the charge sensor has been normalized out of the data as described in Supplementary Note 7. e, f Simulated two-qubit response to simultaneous driving. In this measurement, the right double dot pulse starts 150 ps before the left double dot pulse. We note that the time evolution in this figure occurs near each qubit's anti-crossing, so coherent oscillations persist for longer times than those in Fig. 2.

To measure $M_{\text {exp }}$, we follow the pulse sequences shown in Fig. $4 \mathrm{~b}$ to prepare each input state $\{L R, L L, R R, R L\}$. We then measure the resulting output after application of an additional driving pulse of length $\tau_{t}$ on our target qubit. As discussed in the Methods section, the charge sensor dedicated to the control qubit measures both qubits simultaneously. To account for this, we use the calibrated signal from the target qubit's charge sensor to isolate the control qubit response. We then perform a maximum likelihood estimate to ensure positive probabilities ${ }^{29}$. Figure $4 c, d$ shows the results of this measurement.

Selecting $\tau_{t}=74$ ps maximizes the average of the logical state input fidelities (the inquisition $\mathcal{I}^{30}$ ) at a modest value of $\mathcal{I}=63 \%$. At this point, in the $\{L R, L L, R R, R L\}$ basis,

$$
M_{\exp }=\left(\begin{array}{llll}
0.22 & 0.65 & 0.12 & 0.09 \\
0.68 & 0.33 & 0.02 & 0.13 \\
0.03 & 0.02 & 0.73 & 0.32 \\
0.08 & 0.01 & 0.13 & 0.46
\end{array}\right)
$$

which we compare to an ideal conditional $\pi$-rotation

$M_{\pi}=\left(\begin{array}{llll}0 & 1 & 0 & 0 \\ 1 & 0 & 0 & 0 \\ 0 & 0 & 1 & 0 \\ 0 & 0 & 0 & 1\end{array}\right)$.

Notably, the input state that requires the most preparation $(|R L\rangle)$ has a significantly lower fidelity $(46 \%)$ than the other input states. This suggests that state preparation errors are a dominant source of infidelity in our conditional operation, although tunnel coupling noise and state relaxation could also contribute as discussed in Supplementary Note 9.

\section{DISCUSSION}

Although the $74 \mathrm{ps}$ conditional $\pi$-rotation demonstrated here is consistent with a two-qubit CNOT, the $T_{2}^{*}=80 \pm 20 \mathrm{ps}$ dephasing time of the control qubit during its idle step limits any claim of a coherent two-qubit processor. Nevertheless, the $13.5 \mathrm{GHz}$ two- 
(a)

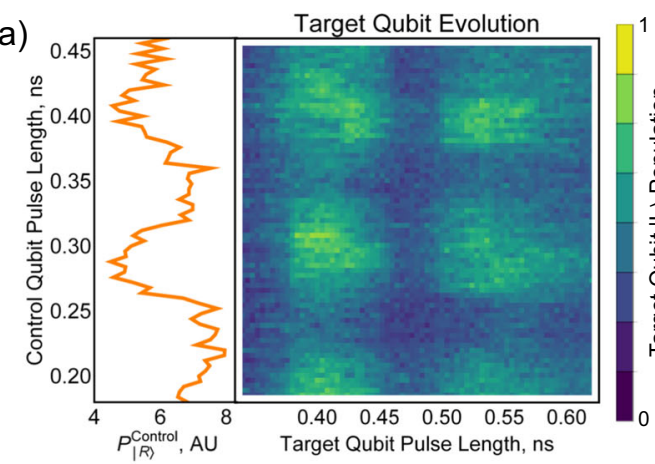

(b)

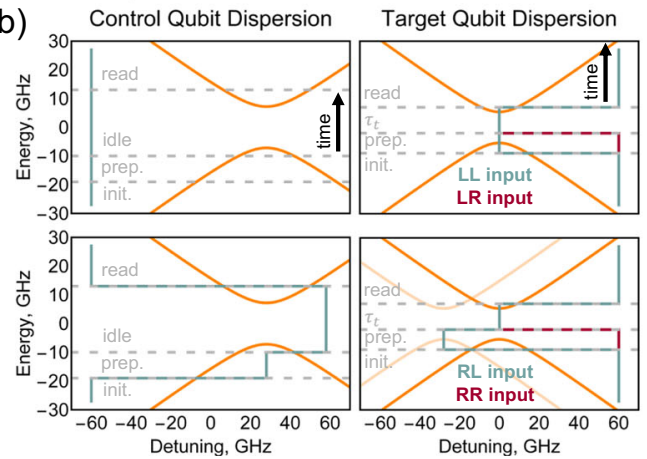

(c)

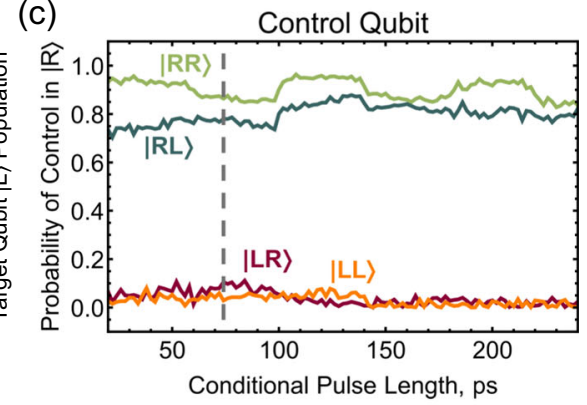

(d)

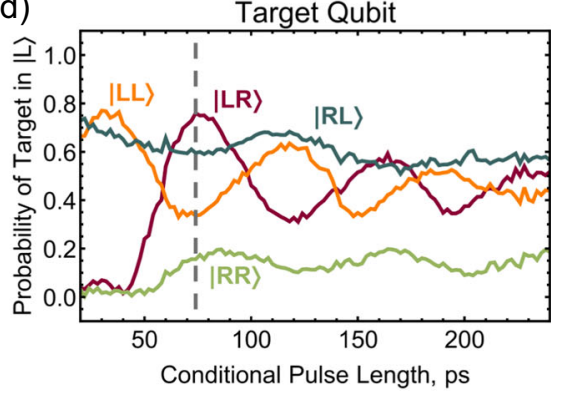

Fig. 4 Conditional qubit evolution. a Evolution of the target qubit conditional on the coherent driving of the control qubit. A onedimensional slice of the control qubit evolution is plotted on the left. $\mathbf{b}$ Dispersions (orange) and pulse sequences (cyan, red) used to measure conditional rotations. The top (bottom) pair of figures was used to prepare two qubit states with the control qubit in $|L\rangle(|R\rangle)$. The different control sequences used to prepare and measure the four input states are color-coded. $\mathbf{c}$, $\mathbf{d}$ Evolution of the four input states in the $\mathbf{c}$ control and $\mathbf{d}$ target qubits. Charge sensor crosstalk has been subtracted from the control qubit data as described in the Methods section.

qubit clockspeed highlights the benefit of using the strong capacitive interaction for inter-qubit coupling. Encoded qubits that have a tunable electric dipole moment such as the QDHQ stand to benefit from this fast gate speed without suffering from dephasing during idle periods. Compared to the charge qubits used in this work, higher fidelity single-qubit operations ${ }^{18}$ and longer coherence times ${ }^{31}$ for the QDHQ could also reduce state preparation errors and enable the extended pulse sequences needed for a multi-qubit processor.

In summary, we have demonstrated correlated and conditional evolution between two capacitively coupled charge qubits. After quantifying the single-qubit coherences, we simultaneously drove coherent rotations in both qubits to demonstrate correlated twoqubit evolution. We then operated in a sequential-driving mode to demonstrate a fast $(74 \mathrm{ps})$ conditional $\pi$-rotation with a modest average fidelity $(63 \%)$ that was likely limited by state-preparation errors. These results represent an important demonstration of the promise capacitive coupling holds for two-qubit interactions in Si/ SiGe double dot qubits.

\section{METHODS}

\section{Crosstalk subtraction}

During our two-qubit measurements, the left charge sensor was sensitive to both the LDD and RDD qubits, whereas the right charge sensor was only sensitive to the RDD qubit dynamics. Supplementary Note 7 provides data demonstrating this crosstalk, which obfuscates the LDD qubit dynamics. A series of appropriate normalization measurements allow us to deconvolve the LDD and RDD signal from the left sensor data.

Since the right sensor only measures RDD qubit dynamics, two normalization measurements are performed for this signal. First, the right sensor is measured after the LDD and RDD qubits have been initialized into $|L\rangle$ and $|R\rangle$, respectively, providing $\mathcal{R}_{\mathrm{LR}}$. Next, the right sensor is measured after the RDD qubit has been pulsed into the $(1,1)$ latched state. This is done by rapidly shifting the RDD to a large negative detuning, delaying at that point until the system has relaxed into $|L\rangle$, and rapidly shifting back to the readout window for latching. This second measurement provides $\mathcal{R}_{\mathrm{LL}}$.

The left charge sensor measures both qubits, so more normalization measurements are required to deconvolve its signal. First, the pulses described in the previous paragraph are repeated, and the left sensor current is monitored. This provides the quantities $\mathcal{L}_{\mathrm{LR}}$ and $\mathcal{L}_{\mathrm{LL}}$. These same measurements are then repeated again with the pulses applied to the LDD qubit instead of the RDD qubit to obtain $\mathcal{L}_{\mathrm{LR}}$ (again) and $\mathcal{L}_{\mathrm{RR}}$. A final normalization measurement applies pulses to both the LDD and the RDD to obtain $\mathcal{L}_{\mathrm{RL}}$.

It is worth noting that our time-averaged measurement technique integrates signal over the entire duty cycle of the pulse sequence. This pollutes our data with signal generated during the manipulation portion of the duty cycle. For all of our measurements, however, the manipulation time is many orders of magnitude shorter than the measurement time and any pollution is negligible. This effect is most significant for the measurements of $\mathcal{R}_{\mathrm{LL}}, \mathcal{L}_{\mathrm{LL}}, \mathcal{L}_{\mathrm{RR}}$, and $\mathcal{L}_{\mathrm{RL}}$ where the manipulation time rises to $\sim 1 \%$ of the total duty cycle.

After obtaining normalization data, qubit measurements are performed to obtain the uncalibrated signal $\mathcal{L}$ and $\mathcal{R}$. Because the right charge sensor only measures the RDD qubit, we can first calibrate $\mathcal{R}$ to obtain the probability the RDD qubit has ended its evolution in state $|L\rangle$ :

$$
P_{|L\rangle}^{\mathrm{RDD}}=\frac{\mathcal{R}-\mathcal{R}_{\mathrm{LR}}}{\mathcal{R}_{\mathrm{LL}}-\mathcal{R}_{\mathrm{LR}}} \text {. }
$$

The left charge sensor measures both qubits simultaneously. To account for this, we first need to determine how the two qubit signals are combined in the charge sensor response. In Supplementary Note 7, we see that the LDD and the RDD both contribute positively to the left sensor signal, and comparing normalization pulses, we find $\mathcal{L}_{\mathrm{RR}}>\mathcal{L}_{\mathrm{RL}}>\mathcal{L}_{\mathrm{LL}}>\mathcal{L}_{\mathrm{LR}}$. Making the assumption of monotonic contributions to the charge sensor signal, we explain this behavior with a LDD signal whose dynamic range depends on the state of the RDD. If the RDD is in $|R\rangle$ then the LDD signal ranges from $\mathcal{L}_{R R}$ to $\mathcal{L}_{\mathrm{LR}}$, whereas with the RDD in $|L\rangle$, the LDD contribution ranges from $\mathcal{L}_{\mathrm{RL}}$ to $\mathcal{L}_{\mathrm{LL}}$. The RDD contribution, however, always ranges from $\left(\mathcal{L}_{\mathrm{LL}}-\mathcal{L}_{\mathrm{LR}}\right)$ to 0 .

To apply this model to our data, we approximate the combined signal by

$$
\mathcal{L}=\mathcal{L}_{\mathrm{LDD}}+\mathcal{L}_{\mathrm{RDD}}
$$


where $\mathcal{L}_{\mathrm{RDD}}\left(\mathcal{L}_{\mathrm{LDD}}\right)$ is the RDD's (LDD's) contribution to the left sensor signal. The calibrated right sensor signal allows us to calculate

$$
\mathcal{L}_{\mathrm{RDD}}=P_{|1\rangle}^{\mathrm{RDD}} \times\left(\mathcal{L}_{\mathrm{LL}}-\mathcal{L}_{\mathrm{LR}}\right) \text {. }
$$

Combining Eqs. (6) and (7) and calibrating with our normalization data, we can then write the probability the LDD qubit has ended its evolution in state $|R\rangle$ as

$P_{|R\rangle}^{\mathrm{LDD}}=\frac{\mathcal{L}-P_{|L\rangle}^{\mathrm{RDD}} \times\left(\mathcal{L}_{\mathrm{LL}}-\mathcal{L}_{\mathrm{LR}}\right)-c_{\text {min }}}{c_{\max }-c_{\min }}$

where

$c_{\text {min }}=\mathcal{L}_{\mathrm{LL}} P_{|L\rangle}^{\mathrm{RDD}}+\mathcal{L}_{\mathrm{LR}}\left(1-P_{|L\rangle}^{\mathrm{RDD}}\right)$

and

$c_{\max }=\mathcal{L}_{\mathrm{RL}} P_{|L\rangle}^{\mathrm{RDD}}+\mathcal{L}_{\mathrm{RR}}\left(1-P_{|L\rangle}^{\mathrm{RDD}}\right)$

define the state-dependent ranges of $\mathcal{L}_{\mathrm{LDD}}$. Notably, applying this procedure to our normalization pulses returns the expected probabilities.

\section{Maximum likelihood estimation}

In Supplementary Note 7, we plot Fig. $4 \mathrm{c}$ with and without the charge sensor crosstalk subtracted. For some portions of these data, this crosstalk removal procedure returns a slightly negative probability. To make sense of this unphysical result, we apply a maximum likelihood estimator (MLE) to our single-qubit states to enforce positivity of the reported probabilities. Because we have assumed separable states in our conditional measurements, applying this MLE at the single-qubit or the two-qubit level provides identical results.

The MLE aims to find the physically-valid density matrix $\rho_{\mathrm{p}}$ that most closely approximates our measured density matrix $\rho_{\text {exp. }}$. Since we can only measure the diagonal elements of $\rho_{\text {exp }}$, we adapt the MLE protocol used by James et al. ${ }^{29}$ to neglect coherences. We constrain $\rho_{\mathrm{p}}$ to be a nonnegative, definite matrix by defining $\rho_{\mathrm{p}}=\hat{T}^{\dagger} \hat{T} / \operatorname{Tr}\left[\hat{T}^{\dagger} \hat{T}\right]$ where

$$
\hat{T}=\left(\begin{array}{cc}
t_{1} & 0 \\
0 & t_{2}
\end{array}\right) \text {. }
$$

We then make the assumption that for each element $\rho_{\exp , i}$ imperfections in our measurements generate a Gaussian probability of measuring the physical value $\rho_{\mathrm{p}, i}$ and the standard deviation of that distribution is approximated by $\sqrt{\rho_{\mathrm{p}, i}}{ }^{29}$. The probability that $\rho_{\mathrm{p}}$ could produce $\rho_{\exp }$ then becomes

$$
P\left(\rho_{\exp }\right)=\frac{1}{N} \prod_{i=1}^{4} \exp \left[\frac{-\left(\rho_{\mathrm{p}, i}-\rho_{\exp , i}\right)^{2}}{2 \rho_{\mathrm{p}, i}}\right]
$$

where $N$ is a normalization constant. Rather than maximizing Eq. (12), we instead maximize its logarithm, which amounts to minimizing the function

$$
L\left(\rho_{\exp }\right)=\sum_{i=1}^{4} \frac{\left(\rho_{\mathrm{p}, i}-\rho_{\exp , i}\right)^{2}}{2 \rho_{\mathrm{p}, i}} .
$$

The diagonal elements of the resulting $\rho_{\mathrm{p}}$ then fill in the columns of $M_{\exp }$ providing the truth table quoted above. In Supplementary Note 7, we plot the results of this MLE process for the control and target qubit data.

\section{DATA AVAILABILITY}

The data that support the findings of this study are available from the corresponding authors upon reasonable request.

Received: 15 March 2020; Accepted: 20 August 2020; Published online: 18 September 2020

\section{REFERENCES}

1. Yoneda, J. et al. A quantum-dot spin qubit with coherence limited by charge noise and fidelity higher than 99.9\%. Nat. Nanotechnol. 13, 102-106 (2018).

2. Brunner, R. et al. Two-qubit gate of combined single-spin rotation and interdot spin exchange in a double quantum dot. Phys. Rev. Lett. 107, 146801 (2011).

3. Veldhorst, M. et al. A two-qubit logic gate in silicon. Nature 526, 410-414 (2015).
4. Zajac, D. M. et al. Resonantly driven cnot gate for electron spins. Science 359, 439-442 (2018).

5. Watson, T. F. et al. A programmable two-qubit quantum processor in silicon. Nature 555, 633-637 (2018).

6. Huang, W. et al. Fidelity benchmarks for two-qubit gates in silicon. Nature 569, 532-536 (2019).

7. Xue, X. et al. Benchmarking gate fidelities in a Si/SiGe two-qubit device. Phys. Rev. $X$ 9, 021011 (2019).

8. He, Y. et al. A two-qubit gate between phosphorus donor electrons in silicon. Nature 571, 371-375 (2019).

9. Sigillito, A. J., Gullans, M. J., Edge, L. F., Borselli, M. \& Petta, J. R. Coherent transfer of quantum information in silicon using resonant swap gates. npj Quantum Inform. 5, 110 (2019).

10. Borjans, F., Croot, X. G., Mi, X., Gullans, M. J. \& Petta, J. R. Resonant microwavemediated interactions between distant electron spins. Nature 577, 195-198 (2020).

11. Li, H.-O. et al. Conditional rotation of two strongly coupled semiconductor charge qubits. Nat. Commun. 6, 8681 (2015).

12. Shulman, M. D. et al. Demonstration of entanglement of electrostatically coupled singlet-triplet qubits. Science 336, 202-205 (2012).

13. Nichol, J. M. et al. High-fidelity entangling gate for double-quantum-dot spin qubits. npj Quantum Inform. 3, 3 (2017).

14. Zajac, D. M., Hazard, T. M., Mi, X., Nielsen, E. \& Petta, J. R. Scalable gate architecture for a one-dimensional array of semiconductor spin qubits. Phys. Rev. Appl. 6, 054013 (2016).

15. Neyens, S. F. et al. Measurements of capacitive coupling within a quadruplequantum-dot array. Phys. Rev. Appl. 12, 064049 (2019).

16. Ward, D. R. et al. State-conditional coherent charge qubit oscillations in a si/sige quadruple quantum dot. npj Quantum Inform. 2, 16032 (2016).

17. Shi, Z. et al. Fast hybrid silicon double-quantum-dot qubit. Phys. Rev. Lett. 108, 140503 (2012).

18. Kim, D. et al. High-fidelity resonant gating of a silicon-based quantum dot hybrid qubit. npj Quantum Inform. 1, 15004 (2015).

19. Frees, A., Mehl, S., Gamble, J. K., Friesen, M. \& Coppersmith, S. N. Adiabatic twoqubit gates in capacitively coupled quantum dot hybrid qubits. npj Quantum Inf. 5, 73 (2019).

20. Angus, S. J., Ferguson, A. J., Dzurak, A. S. \& Clark, R. G. Gate-defined quantum dots in intrinsic silicon. Nano Lett. 7, 2051-2055 (2007).

21. Borselli, M. G. et al. Undoped accumulation-mode si/SiGe quantum dots. Nanotechnology 26, 375202 (2015).

22. Tracy, L. A. et al. Single shot spin readout using a cryogenic high-electronmobility transistor amplifier at sub-kelvin temperatures. Appl. Phys. Lett. 108, 063101 (2016).

23. Studenikin, S. A. et al. Enhanced charge detection of spin qubit readout via an intermediate state. Appl. Phys. Lett. 101, 233101 (2012).

24. Harvey-Collard, P. et al. High-fidelity single-shot readout for a spin qubit via an enhanced latching mechanism. Phys. Rev. X 8, 021046 (2018).

25. Fujisawa, T., Hayashi, T., Cheong, H., Jeong, Y. \& Hirayama, Y. Rotation and phaseshift operations for a charge qubit in a double quantum dot. Phys. E: Low. Dimens. Syst. Nanostruct. 21, 1046-1052 (2004).

26. Mi, X., Kohler, S. \& Petta, J. R. Landau-zener interferometry of valley-orbit states in si/sige double quantum dots. Phys. Rev. B 98, 161404 (2018).

27. Dial, O. E. et al. Charge noise spectroscopy using coherent exchange oscillations in a singlet-triplet qubit. Phys. Rev. Lett. 110, 146804 (2013).

28. Vandersypen, L. M. K. \& Chuang, I. L. Nmr techniques for quantum control and computation. Rev. Mod. Phys. 76, 1037-1069 (2005).

29. James, D. F. V., Kwiat, P. G., Munro, W. J. \& White, A. G. Measurement of qubits. Phys. Rev. A 64, 052312 (2001).

30. White, A. G. et al. Measuring two-qubit gates. J. Opt. Soc. Am. B 24, 172-183 (2007).

31. Thorgrimsson, B. et al. Extending the coherence of a quantum dot hybrid qubit. npj Quantum Inform. 3, 32 (2017).

\section{ACKNOWLEDGEMENTS}

This research was sponsored in part by the Army Research Office (ARO) under Grant Number W911NF-17-1-0274 and by the Vannevar Bush Faculty Fellowship program under ONR grant number N00014-15-1-0029. We acknowledge the use of facilities supported by NSF through the UW-Madison MRSEC (DMR-1720415). The views and conclusions contained in this document are those of the authors and should not be interpreted as representing the official policies, either expressed or implied, of the Army Research Office (ARO), or the U.S. Government. The U.S. Government is authorized to reproduce and distribute reprints for Government purposes notwithstanding any copyright notation herein. 


\section{AUTHOR CONTRIBUTIONS}

E.R.M., S.F.N., and M.A.E. performed the measurements and analysis. S.F.N., E.R.M., J.P. D., and N.H. designed and fabricated the device. B.T., J.C., M.P., S.F.N., and E.R.M. developed experimental protocols and hardware. L.F.E. grew the heterostructure material. E.R.M., M.A.E., M.F., and S.N.C. wrote the manuscript with feedback from all the authors. M.A.E. initiated the project and supervised the work with M.F. and S.N.C.

\section{COMPETING INTERESTS}

The authors declare no competing interests.

\section{ADDITIONAL INFORMATION}

Supplementary information is available for this paper at https://doi.org/10.1038/ s41534-020-00314-w.

Correspondence and requests for materials should be addressed to M.A.E.

Reprints and permission information is available at http://www.nature.com/ reprints
Publisher's note Springer Nature remains neutral with regard to jurisdictional claims in published maps and institutional affiliations.

(i) Open Access This article is licensed under a Creative Commons Attribution 4.0 International License, which permits use, sharing, adaptation, distribution and reproduction in any medium or format, as long as you give appropriate credit to the original author(s) and the source, provide a link to the Creative Commons license, and indicate if changes were made. The images or other third party material in this article are included in the article's Creative Commons license, unless indicated otherwise in a credit line to the material. If material is not included in the article's Creative Commons license and your intended use is not permitted by statutory regulation or exceeds the permitted use, you will need to obtain permission directly from the copyright holder. To view a copy of this license, visit http://creativecommons. org/licenses/by/4.0/.

(c) The Author(s) 2020 